\title{
Contributions to the smut fungi of Africa. 7. First records of Tilletia brachypodii-ramosi and Ustilago constantineanui
}

\section{Teodor T. Denchev ${ }^{1,2 *} \&$ Cvetomir M. Denchev ${ }^{1,2}$}

${ }^{1}$ Institute of Biodiversity and Ecosystem Research, Bulgarian Academy of Sciences, 2 Gagarin St., 1113 Sofia, Bulgaria

${ }^{2}$ IUCN SSC Rusts and Smuts Specialist Group

Received 1 December 2021 / Accepted 13 December 2021 / Published 14 December 2021

Denchev, T.T. \& Denchev, C.M. 2021. Contributions to the smut fungi of Africa. 7. First records of Tilletia brachypodii-ramosi and Ustilago constantineanui. - Mycobiota 11: 11-16. doi: 10.12664/ mycobiota.2021.11.02

\begin{abstract}
Two poorly known smut fungi are recorded for the first time from Africa: Tilletia brachypodii-ramosi on Brachypodium retusum (from Morocco) and Ustilago constantineanui on Sporobolus schoenoides (from Algeria). Descriptions and illustrations are provided for these taxa.

Key words: Africa, Algeria, Brachypodium retusum, Morocco, smut fungi, Sporobolus schoenoides, taxonomy, Tilletia brachypodii-ramosi, Ustilago constantineanui
\end{abstract}

\section{Introduction}

During an examination of grass specimens in the herbarium of the National Museum of Natural History, Paris (P), some interesting smut fungi were found, two of which, Tilletia brachypodii-ramosi and Ustilago constantineanui, are reported here for the first time from Africa (cfr Vánky et al. 2011).

\section{Material and methods}

Dried specimens from the herbarium of the National Museum of Natural History, Paris were examined under light microscope (LM) and scanning electron microscope (SEM). For

\footnotetext{
*Corresponding author: e-mail: ttdenchev@gmail.com
} 
LM observations and measurements, spores and sterile cells were mounted in lactoglycerol solution ( $\mathrm{w}: \mathrm{la}: \mathrm{gl}=1: 1: 2$ ) on glass slides, gently heated to boiling point to rehydrate the spores and sterile cells, and then cooled. The measurements of spores and sterile cells are given as min-max (extreme values) (mean \pm 1 standard deviation). For SEM, spores and sterile cells were attached to specimen holders by double-sided adhesive tape and coated with gold using an ion sputter coater. The surface structure of spores and sterile cells was observed and photographed at $10 \mathrm{kV}$ accelerating voltage using a Hitachi SU3500 scanning electron microscope. The descriptions below are based entirely on the specimens examined. The shapes of spores and sterile cells are arranged in descending order of frequency.

\section{Taxonomy}

Tilletia brachypodii-ramosi H. Zogg, Ber. Schweiz. Bot. Ges. 77: 53, 1967. - Holotype on Brachypodium retusum (Pers.) P. Beauv. (as 'B. ramosum (L.) Roem. \& Schult.') France, Vaucluse Department, Mont Ventoux, 8 km from Malaucène, alt. 850 m, 22 May 1962, H. Zogg, s.n. (ZT).

Figs 1-5

Infection systemic. Sori in leaves as long, swollen striae, initially covered by the epidermis that later ruptures disclosing a very dark reddish brown, powdery mass of spores and sterile cells. Sterile cells subglobose to irregular, often collapsed, usually smaller than spores, (10.5-)11.5-18.5(-20.5) × (8.5-)10-15(-16.5) $(15.5 \pm 2.2 \times 13.2 \pm 1.8) \mu \mathrm{m}(\mathrm{n}$ $=50$ ), subhyaline to pale yellowish; cell wall two-layered, (1.1-)1.3-1.7(-2.0) $\mu \mathrm{m}$ thick. In SEM, smooth to rugulose. Spores subglobose, broadly ellipsoidal, globose or slightly irregular, (18-)19-23(-24) × (17-)18-22(-23) $(20.5 \pm 1.1 \times 19.4 \pm 1.3) \mu \mathrm{m}(\mathrm{n}=100)$, medium reddish brown to dark reddish brown, reticulate; spore wall 1.6-2.3(-2.6) $\mu \mathrm{m}$ thick (including reticulum); meshes $7-9(-10)$ per spore diameter, polyhedral or irregular, (0.6-)1.0-3.5(-4.3) $\mu \mathrm{m}$ long; muri 23-31 on equatorial circumference, in optical median view subacute or blunt, $0.6-1.4(-1.7) \mu \mathrm{m}$ high; sometimes covered by a thin hyaline sheath; sometimes with a papilla. In SEM, interspaces sometimes with a hemispherical protuberance.

Specimen examined - On Brachypodium retusum (Pers.) P. Beauv.: MOROCCO, the High Atlas Range, above Seksaoua, alt. 1200-1600 m, 26-27 May 1871, leg. J. Ballz, Iter Maroccanum 1871 (P-P02627423; as 'B. ramosum').

Distribution - On Poaceae: Brachypodium retusum; Europa (France, Spain, and Croatia) and Africa (Morocco).

Two species of Tilletia form sori in leaves of Brachypodium: T. olida (Riess) J. Schröter on Brachypodium pinnatum (L.) P. Beauv. and B. sylvaticum (Huds.) P. Beauv., and Tilletia brachypodii-ramosi on Brachypodium retusum (Zogg 1967; Vánky 1994, 2011). Tilletia brachypodii-ramosi is distinguished from T. olida by having higher muri, 0.6-1.5(-2.5) $\mu \mathrm{m}$ high vs. $0.3-1.0(-1.3) \mu \mathrm{m}$ high for T. olida.

Tilletia brachypodii-ramosi is known from France, Spain (mainland Spain and the Balearic Islands), and Croatia (Jaap 1916, as 'T. olida'; Guyot et al. 1955, as 'T. olida'; Zogg 1967; Vánky 1979, 1994, 2005, 2011; Ivić et al. 2013). This smut fungus is reported here for the first time from Morocco. 


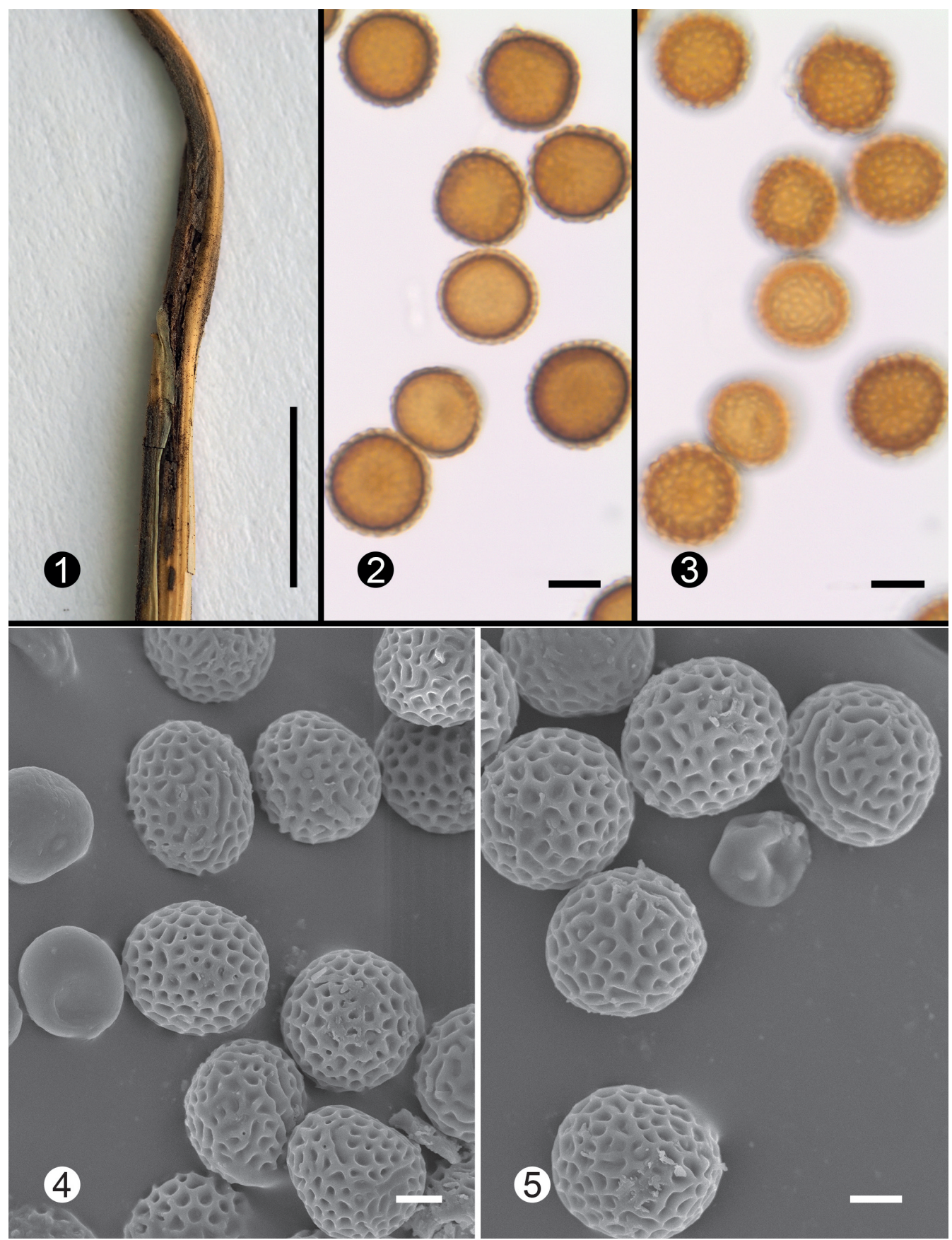

Figs 1-5. Tilletia brachypodii-ramosi on Brachypodium retusum (P-P02627423). 1. Habit. 2, 3. Spores in LM (in median and surface view, respectively). 4, 5. Spores and sterile cells in SEM. Scale bars: $1=0.5 \mathrm{~cm}, 2,3=10 \mu \mathrm{m}, 4,5=5 \mu \mathrm{m}$ 


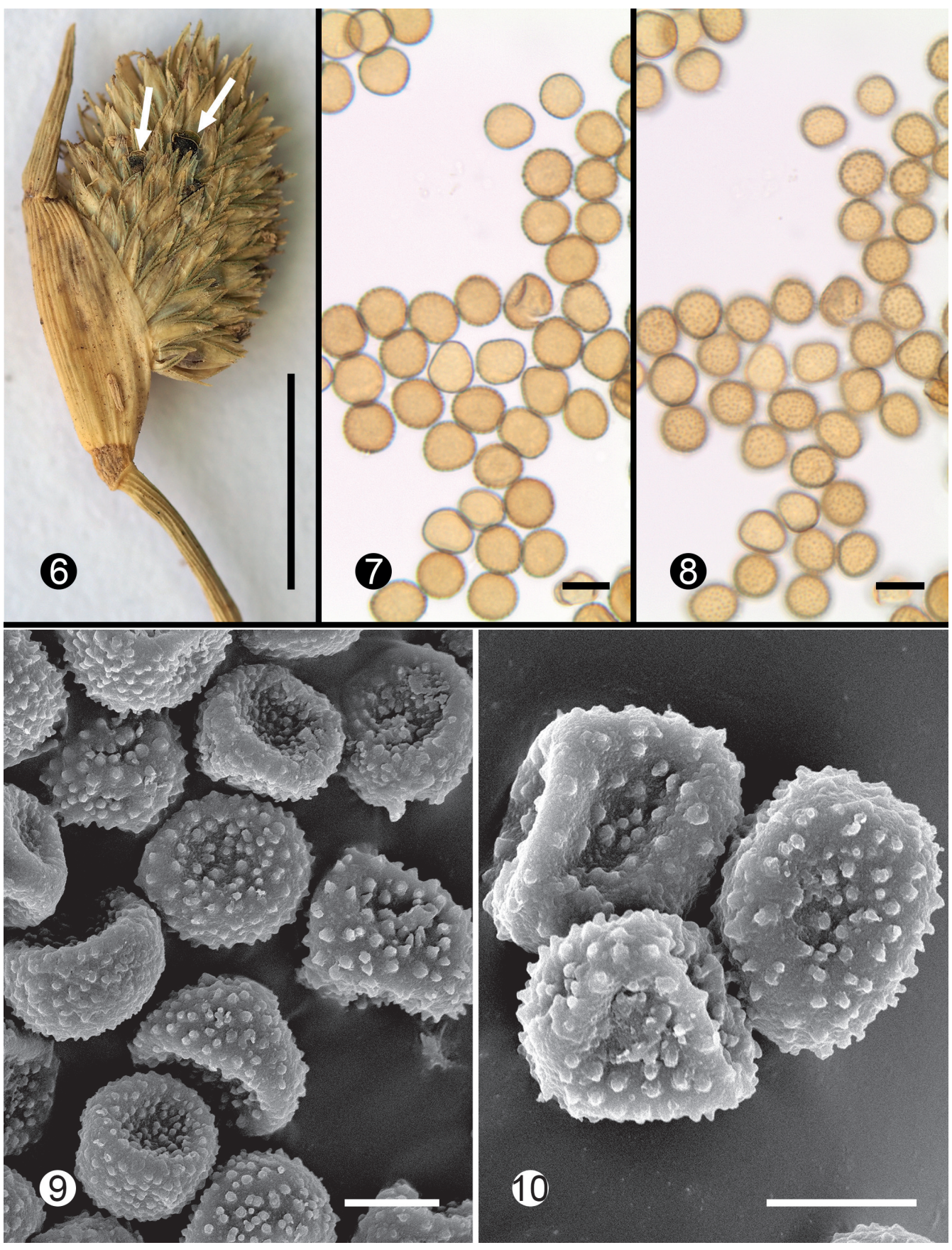

Figs 6-10. Ustilago constantineanui on Sporobolus schoenoides (P-P02655721). 6. Habit; arrows show sori. 7, 8. Spores in LM (in median and surface view, respectively). 9, 10. Spores in SEM. Scale bars: $6=0.5 \mathrm{~cm}, 7,8=10 \mu \mathrm{m}, 9,10=5 \mu \mathrm{m}$ 
Ustilago constantineanui (Săvul.) Vánky, Symb. Bot. Upsal. 24(2): 201, 1985. ĐSphacelotheca constantineanui Săvul., Bul. Şti. Acad. Republ. Populare Române 3: 214, 1951. — Holotype on 'Alopecurus aequalis Sobol.' (misidentified Sporobolus alopecuroides, det. K. Vánky, 1985: 201), Romania, Giurgiu County, Bolintin, 4 Oct 1918, leg. M. Brândză, s.n. (BUCM).

Figs 6-10

= Ustilago heleochloae Vánky \& Gönczöl, Bot. Not. 131: 247, 1978. — Holotype on Sporobolus schoenoides (as 'Heleochloa schoenoides'), Hungary, Budapest, near Pestlörinc, $47^{\circ} 30^{\prime} \mathrm{N}, 19^{\circ} 03^{\prime} \mathrm{E}$, alt. 130 m, 10 Oct 1977, J. Gönczöl, s.n. (HUV 6514, isotypes in BP, S, and Vánky, Ustilag., no. 313) (syn. by Vánky 1985: 201).

Infection local. Sori in single ovaries of infected plant, inconspicuous, hidden by the glumes, broadly ellipsoidal, ellipsoidal, subglobose or lemon-shaped, 1.0-2.2 × 0.8-1.8 $\mathrm{mm}$, covered by a thin, greenish brown pericarp that later ruptures exposing a powdery, dark reddish brown mass of spores. Spores single, subglobose, broadly ellipsoidal, irregular, ellipsoidal, globose or ovoid, (8.5-)9.5-13(-14) x (7.5-)8.5-10.5(-11.5) $(11.0 \pm 1.0 \times$ $9.7 \pm 0.7) \mu \mathrm{m}(\mathrm{n}=100)$, medium yellowish brown; wall $0.9-1.3 \mu \mathrm{m}$ thick, echinulateverrucose, ornaments up to $0.5(-0.6) \mu \mathrm{m}$ high. In SEM, spore wall punctate between the ornaments.

Specimen examined - On Sporobolus schoenoides (L.) P.M. Peterson: ALGERIA, SIDI BEL ABBÈS PROVINCE, near Sidi Bel Abbès, 25 Aug 1873, leg. A. Warion, Plantae Atlanticae Selectae, no. 96 (P-P02655721; as 'Crypsis schoenoides').

Distribution - On Poaceae: Sporobolus aculeatus (L.) P.M. Peterson (Crypsis aculeata (L.) Aiton), S. alopecuroides (Piller \& Mitterp.) P.M. Peterson (Crypsis alopecuroides (Piller \& Mitterp.) Schrad.), S. schoenoides (Crypsis schoenoides (L.) Lam., Heleochloa schoenoides (L.) Host ex Roem.); Europa (Hungary and Romania) and Africa (Algeria).

Ustilago constantineanui is known only from Hungary and Romania (Săvulescu 1951, as 'Sphacelotheca constantineanui; Vánky \& Gönczöl 1978, as 'Ustilago heleochloae'; Vánky 1980, as 'Ustilago heleochloae'; Vánky 1985a, b, 1994, 2011). This smut fungus is reported here for the first time from Algeria.

Acknowledgements. This study was supported by the Bulgarian Science Fund (Grant no. KP-06-N51/10/16.11.2021). The visit to the herbarium of the National Museum of Natural History, Paris was carried out in the framework of the SYNTHESYS Project (http://www. synthesys.info/) financed by European Community Research Infrastructure Action under the FP7 “Capacities” Program (Grant no. FR-TAF-6628).

\section{References}

Guyot, L., Malençon, G. \& Massenot, M. 1955. Contribution à I'étude des Ustilaginales parasites du Bassin Méditerranéen Occidental (Afrique du Nord et Péninsule ibérique). - Revue de Pathologie Végétale et d'Entomologie Agricole de France 34: 181-216.

Ivic, D., Sever, Z., Scheuer, C. \& Lutz, M. 2013. A checklist of smut fungi in Croatia. - Mycotaxon 121: 499. Jaap, O. 1916. Beiträge zur Kenntnis der Pilze Dalmatiens. - Annales Mycologici 14: 1-44. 
Săvulescu, T. 1951. Noutăţi din micoflora Republicii Populare Române. - Buletin Stiinţific, Academia Republicii Populare Române, Secţiunea de ştiinţe biologice, agronomice, geologice şi geografice 3: 211-227 + Plates 1-3.

Vánky, K. 1979. Ustilaginales. Fasc. VI-X (No. 126-250). - Publications from the Herbarium of University of Uppsala, Sweden 3: 1-32.

Vánky, K. 1980. Ustilaginales. Fasc. XI-XIII (No. 251-325). - Publications from the Herbarium of University of Uppsala, Sweden 5: 1-25.

Vánky, K. 1985a. K. Vánky, Ustilaginales exsiccate. Fasc. XIX-XX (No. 451-500). - Publications from the Herbarium Ustilaginales Vánky 1: 1-17.

Vánky, K. 1985b. Carpathian Ustilaginales. - Symbolae Botanicae Upsalienses 24(2): 1-309.

Vánky, K. 1994. European smut fungi. Gustav Fischer Verlag, Stuttgart, Jena, New York.

Vánky, K. 2005. European smut fungi (Ustilaginomycetes p.p. and Microbotryales) according to recent nomenclature. - Mycologia Balcanica 2: 169-177.

https://doi.org/10.5281/zenodo.2546967

Vánky, K. 2011['2012']. Smut fungi of the world. APS Press, St. Paul, Minnesota.

Vánky, K. \& Gönczöl, J. 1978. Two new species of Ustilaginales from Hungary. - Botaniska Notiser 131: 247-250.

Vánky, K., Vánky, C. \& Denchev, C.M. 2011. Smut fungi in Africa - a checklist. - Mycologia Balcanica 8: $1-77$. https://doi.org/10.5281/zenodo.2550336

Zogg, H. 1967. Über die Sporenkeimung von Tilletia olida (Riess ap. Rab.) Schröter und Tilletia brachypodiiramosi n. sp. - Berichte der Schweizerischen Botanischen Gesellschaft 77: 49-56 + Plate. 